\title{
Rectal perforation in a 42-year-old woman due to Henoch-Schönlein purpura: a case report
}

\author{
F. Almassinokiani', A. Mehdizadeh Kashi², A. Musavi³,S. Khodaverdi², \\ K. Tahermanesh ${ }^{2}$, S. Ariana ${ }^{3}$ \\ ${ }^{1}$ Department of Minimally Invasive Gynecology Surgery, Minimally Invasive Surgery Research Center, \\ Iran University of Medical Sciences (IUMS), Tehran, Iran; ${ }^{2}$ Department of Minimally Invasive Gynecology \\ Surgery, Endometriosis Research Center, Iran University of Medical Sciences (IUMS), Tehran, Iran; \\ ${ }^{3}$ Department of Obstetrics \& Gynecology, Iran University of Medical Sciences (IUMS), Tehran, Iran
}

\begin{abstract}
SUMMARY
Henoch-Schönlein purpura (HSP) is caused by deposition of IgA-containing immune complexes within the blood vessels. HSP mostly occurs in children and is less common in adults. In addition to palpable purpura, arthritis or arthralgia, renal disease and abdominal pain, gastrointestinal symptoms occur in $50 \%$ of children. These can be caused by gastrointestinal hemorrhage, bowel ischemia and necrosis, intussusception, and bowel perforation. In adults, intussusception is less frequent than in children.

We report a 42-year-old woman referred to our hospital with acute abdominal pain. After laboratory and radiologic examinations, laparatomy was performed which revealed rectal perforation due to HSP.

HSP is a multisystem disorder. Abdominal pain in patients with HSP may herald a serious gastrointestinal problem also in adult patients.
\end{abstract}

Key words: Henoch-Schönlein purpura; Bowel perforation; Abdominal pain; Rectal perforation.

Reumatismo, 2017; 69 (3): 131-133

\section{INTRODUCTION}

$\mathrm{H}$ enoch-Schönlein purpura (HSP), also called $\operatorname{IgA}$ vasculitis, is caused by deposition of $\operatorname{IgA}$-containing immune complexes within the blood vessels (1) and its characteristic finding is leukocytoclastic vasculitis. HSP mostly occurs in children between the ages of 3 and 15 years (2) and is less common in adults (3). The incidence of 3.4 to 14.3 per million was reported for HSP in adults (4). HSP manifestations in children include palpable purpura, arthritis or arthralgia, renal disease and abdominal pain (5). Gastrointestinal symptoms like nausea, vomiting, abdominal pain, and transient paralytic ileus occur in $50 \%$ of the children and the findings are: gastrointestinal hemorrhage; bowel ischemia and necrosis; intussusception, and bowel perforation (6). In adults, in addition to the above-mentioned manifestations, there are two main differences: intussusception is rare and the risk of renal disease is increased (7). The diagnosis of HSP is usually based on clinical manifestations, especially palpable purpura in the lower extremities and a biopsy of the involved organ (e.g., skin or kidney) in those who have unusual presentations. There is no specific diagnostic laboratory test (8). Imaging is commonly performed on patients with abdominal pain. Abdominal radiography may show dilatation of the bowel loops; it may demonstrate peritoneal fluid, hematomas, intussusception and an increased bowel wall thickness (9). We report a rare case of rectal perforation due to HSP in a 42-year-old woman.

\section{CASE REPORT}

A 42-year-old woman with chief complaint of abdominal pain, was referred to Rasool-e-Akram hospital in February 2015. Her pain had started gradually a week before in the hypogastric region re-
Corresponding author Sepideh Khodaverdi

Department of Obstetrics \& Gynecology, Minimally Invasive Gynecology Surgery, Endometriosis Research Center,

Iran University of Medical Science (IUMS) Tehran, Sattar Khan st., Niyayesh st., Hazrat Rasoul-e Akram Hospital, Gynecology and

Obstetrics ward, Tehran, Iran. E-mail: research.rasoul@gmail.com 
ferring to her flanks and back. The pain was exacerbated on the day of the visit. The patient was weak and lethargic and had nocturnal sweating, nausea, anorexia and vomiting. The pain increased with defecation and she had a green foulsmelling discharge of her rectum during defecation for 25 days. The patient stated that the feces diameter had reduced in the last week. She had been diagnosed with HSP three months before and had been taking prednisolone $5 \mathrm{mg}$ three times a day for two months and azathioprine 50 $\mathrm{mg}$ daily for 8 days. HSP diagnosis was made by skin biopsy performed because of hyperpigmented skin lesions. Upon physical examination, her general condition was good. Her vital signs were stable (blood pressure $=110 / 70 \mathrm{mmHg}$, respiratory rate $=20$ per minute and pulse rate $=90$ beats $/ \mathrm{min}$ ) but she had a low grade fever on the admission day $\left(38.5^{\circ} \mathrm{C}\right)$. The abdomen was soft, without any distention and resistance and showed tenderness in the hypogastric region (left lower quadrant). Heart and lung examinations were normal. She was divorced and she had not had any sexual intercourse for two years. Mild vaginal discharge was seen at al the vaginal examination without bleeding. The left adnex was tender and no mass could be palpated. Laboratory data on the admission day were as follows: hemoglobin $(\mathrm{Hb}) 128 \mathrm{~g} / \mathrm{L}$; white blood cell (WBC) count $18,700 \mu \mathrm{L}$ with an increased neutrophil percentage $(91.2 \%)$; hematocrit $40.6 \%$; platelet count $470,000 / \mathrm{L}$; erythrocyte sedimentation rate $(E S R) 59 \mathrm{~mm} / 1^{\text {st }}$ $\mathrm{h}$; C-reactive protein (CRP) $96 \mathrm{mg} / \mathrm{L}$; blood urea nitrogen (BUN) $9 \mathrm{mg} / \mathrm{dL}$; serum creatinine $(\mathrm{Cr}) 1.3 \mu \mathrm{mol} / \mathrm{L}$; serum sodium (Na) $139 \mathrm{mmol} / \mathrm{L}$; serum potassium $(\mathrm{K}) 4.9 \mathrm{mmol} / \mathrm{L}$; urine protein, ketone, glucose and culture were negative; stool exam was negative for parasite and occult blood. Abdominal and pelvic computed tomography (CT) demonstrated no mass or para-aortic adenopathy; chest $\mathrm{x}$ ray in the standing position was normal. At abdominal and pelvic ultrasonography at the admission time, the right ovary was enlarged $(5.5 \times 5.5 \mathrm{~cm})$ and contained mul- tiple cystic structures with thick walls and no septation - the biggest one was inside the right ovary $(3.4 \times 2.9 \mathrm{~cm})$. The size of the left ovary was $2 \times 3.2 \mathrm{~cm}$ with normal echo. No free fluid in the abdomen and pelvis was reported. The color Doppler sonography of the right ovary was normal and without any evidence of torsion. Among ovarian tumor markers, CA 125 was as high as $100 \mathrm{U} / \mathrm{ml}$. Serum $\beta$-hCG was checked to rule out ectopic pregnancy (EP) and was negative. After stabilization of the patient's condition and symptomatic treatment, she underwent an elective laparotomy for the presumptive diagnosis of ovarian tumor. Upon opening the abdomen, foul-smelling liquid was detected on the surface of the omentum, which was sent for culture; both ovaries were enlarged and were attached to the bowel and omentum from behind and no cystic or solid lesion was observed; the posterior side of the uterus was perforated and contained pus that was adherent to the bowel. She then underwent a total abdominal hysterectomy and bilateral oophorectomy. The anterior-superior part of rectum was perforated and had a stiff swelling necrosis; appendecectomy was performed by surgeons due to suspicious appearance of omentum and appendix, colostomy was placed and the abdomen was washed with 5 litres of warm saline solution. The culture of abdominal liquid showed Escherichia coli.

\section{DISCUSSION}

HSP is known as a multisystem disorder affecting the skin, gastrointestinal tract, joints, and kidneys, but rarely reported in the nervous system, lung, heart, and genitourinary tract (4). More than $90 \%$ of patients with HSP are children. One of the most common symptoms of the gastrointestinal involvement is abdominal pain (4), as seen in the present case. The most common intra-abdominal complication which needs surgical management is intussusception (with incidence of 3.5\%) (1), but in adults intussusception is rare and renal involvement (ESRD) at al increased risk (2). Less 
frequent reasons for surgery are hemorrhage, necrosis and bowel perforation. Perforation results from vasculitis leading to bowel necrosis (3). In our case the anterior and superior side of the rectum had a stiff swelling necrosis. Treatment with glucocorticoids increases the risk of perforation, usually in the second week of therapy (6). Our patient had been taking prednisolone $5 \mathrm{mg}$ thrice a day for about 2 months. The highest incidence of HSP has been reported in Asians (10). Knowing that bowel perforation in children with HSP usually occurs due to intussusception and is in the small intestine, we report a rare case of HSP in a 42-year old female with rectal perforation who was admitted with abdominal pain and pus discharge during defecation. Terminal ileitis has been reported rarely in the literature $(4,11,12)$. Crohn disease and PID were the other differential diagnosis in this patient but since, according to her history, about 9 months ago she divorced and did not have any subsequent sexual activity, PID was ruled out.

\section{CONCLUSIONS}

HSP is a multisystem disorder. Abdominal pain in patients with HSP may herald a serious gastrointestinal problem also in adult patients. Appropriate diagnostic tests should be performed to rule out severe complications, such as intestinal perforation.

Acknowledgements: the authors wish to thank Rasoul-e-Akram Hospital Clinical Research Development Center, Iran University of Medical Science for technically supported implementation of the project.

Conflict of interest: the authors declare no conflict of interest.

\section{REFERENCES}

1. Jennette JC, Falk RJ, Bacon PA, et al. 2012 revised International Chapel Hill Consensus Conference Nomenclature of Vasculitides. Arthritis Rheum. 2013; 65: 1.

2. Gardner-Medwin JM, Dolezalova P, Cummins C, Southwood TR. Incidence of Henoch-Schönlein purpura, Kawasaki disease, and rare vasculitides in children of different ethnic origins. Lancet. 2002; 360: 1197.

3. Blanco R, Martínez-Taboada VM, Rodríguez-Valverde V, et al. Henoch-Schönlein purpura in adulthood and childhood: two different expressions of the same syndrome. Arthritis Rheum. 1997; 40: 859.

4. Fan Z, Tian X, Pan J, et al. Terminal Ileitis Induced by Henoch-Schonlein purpura that presented as acute appendicitis: a case report. Medicine. 2015; 94: e492.

5. Trapani S, Micheli A, Grisolia F, et al. Henoch Schonlein purpura in childhood: epidemiological and clinical analysis of 150 cases over a 5-year period and review of literature. Semin Arthritis Rheum. 2005; 35: 143.

6. Chang WL, Yang YH, Lin YT, Chiang BL. Gastrointestinal manifestations in HenochSchönlein purpura: a review of 261 patients. Acta Paediatr. 2004; 93: 1427.

7. Pillebout E, Thervet E, Hill G, et al. HenochSchönlein purpura in adults: outcome and prognostic factors. J Am Soc Nephrol. 2002; 13: 1271 .

8. McCarthy HJ, Tizard EJ. Clinical practice: diagnosis and management of Henoch-Schönlein purpura. Eur J Pediatr. 2010; 169: 643.

9. Choong CK, Beasley SW. Intra-abdominal manifestations of Henoch-Schönlein purpura. J Paediatr Child Health. 1998; 34: 405.

10. Sohagia AB, Gunturu SG, Tong TR, et al. Henoch-Schonlein purpura - a case report and review of the literature. Gastroenterol Res Pract. 2010; 2010: 597648.

11. Kanik A, Kose E, Baran M, et al. HenochSchonlein purpura in two pediatric patients presenting as terminal ileitis. Dig Dis Sci. 2015; 60: 269-71.

12. Al-Toma AA, Brink MA, Hagen EC. Henoch-Schönlein purpura presenting as terminal ileitis and complicated by thrombotic microangiopathy. Eur J Intern Med. 2005; 7: 510-2. 